\title{
Betaine adduct of N-heterocyclic carbene and carbodiimide, an efficient ligand to produce ultra-small ruthenium nanoparticles ${ }^{\dagger}$
}

Cite this: DOI: $10.1039 / \times 0 \times x 00000 x$

Received 00th January 2012, Accepted 00th January 2012

DOI: $10.1039 / \times 0 \times x 00000 x$
L. M. Martínez-Prieto, ${ }^{a}$ C. Urbaneja,${ }^{b}$ P. Palma,${ }^{b}$ J. Cámpora, ${ }^{* b}$ K. Philippot ${ }^{* a}$ and B. Chaudret $* c$

www.rsc.org/

The betaine adduct of $\mathrm{N}$-heterocyclic carbene and carbodiimide ( $\mathrm{ICy}^{\left.\cdot{ }^{(p-t o l)} \mathrm{NCN}\right)}$ was found to be a very efficient ligand to prepare very small $(1-1.3 \mathrm{~nm})$ ruthenium nanoparticles (RuNPs). The coordination of the ligand on the metal surface takes place through the carbodiimide moiety. The resulting RuNPs led to decarbonylation of THF and showed a size selectivity for styrene hydrogenation.

Particles of metals at the nanometer scale, namely nanoparticles (NPs), are known to display interesting physical and chemical properties and this confers them a high potential for application in diverse areas like catalysis or others. ${ }^{1}$ For both physical and chemical aspects, a lot of changes are expected to occur at the frontier of the molecular and the solid states where nanoparticles are situated. But to be able to explore these changes and to investigate the resulting properties, it is necessary to selectively produce very small and well-controlled nano-objects. Thus, a main objective in the field is to develop appropriate tools to produce well-defined objects at the nanometer-size. ${ }^{2}$ If methods exist for synthesizing large molecular complexes (clusters) and for growing the particles with a small mean diameter, ${ }^{3}$ it is very difficult to control the size growth in the range of e.g. $1 \mathrm{~nm}$. In our group, we have been working for a long time on the synthesis of RuNPs. ${ }^{4}$ Typical sizes are in the range 1-4 $\mathrm{nm}$ (with a great majority around 1.5-1.8 $\mathrm{nm}$ ) depending on the stabilizer used for their preparation. Until now, it appeared to us difficult to obtain RuNPs smaller than $1 \mathrm{~nm}$ although our favourite ruthenium precursor, namely (1,5-cyclooctadiene)(1,3,5cyclooctatriene)ruthenium $(0)[\mathrm{Ru}(\mathrm{COD})(\mathrm{COT})]$, is very easy to decompose in mild reaction conditions which can make the nucleation step more favourable than the growth of NPs. Thus, the search for effective ligands to stop the NP growth at a desired size like $1 \mathrm{~nm}$ or less, constitutes one of our goals. From previous results, we know that strongly coordinating ligands can limit the growth of the particles (vide supra). As N-heterocyclic carbenes showed a very high ability to coordinate at NP surface (both $\mathrm{Ru}$ and $\mathrm{Pt})^{5}$ due to their specific electron donor properties, we considered the use of more complex ligands derived from NHC-carbenes. More precisely, NHeterocyclic carbenes (NHCs) form stable zwitterionic adducts (betaines) with carbodiimides (NCN). The coordination chemistry of such NHC-NCN betaines has not been yet investigated, but they combine a number of properties that can make them valuable ligands for NP synthesis. For example, their configuration can be assimilated to that of carboxylate or amidinate ligands but displaying an overall electrically neutral character. However, owing to their zwitterionic structure, the $\mathrm{N}$ atoms of NHC-NCN compounds are strongly basic and may coordinate to transition metals more strongly than typical electrically neutral ligands do. In addition, they have a modular design that allows easy variations of the pending groups linked to the nitrogen atoms which may open new routes for controlling the surface properties of MNPs. Moreover, depending on the nature of the ionic moieties, these betaines can show different properties and lead to different applications in organic synthesis and catalysis. ${ }^{6}$ Thus, betaines are ligands which offer both a zwitterionic character and a large steric protection due to the four arms they contain. They could be ideal for lowering NP rate of growth, thus favouring nucleation over growth and therefore for producing smaller nanoparticles. With this aim we looked at the synthesis of RuNPs using the betaine ligand 1,3-dicyclohexylimidazolium-2- di-ptolylcarbodiimide (ICy.(p-tol) $\mathrm{NCN}$ ) as stabilizer in different $\mathrm{Ru} /$ ligand ratios. The coordination mode of this novel ligand at the ruthenium surface as well as its location and the presence of extended faces at RuNP surface were determined by NMR. Moreover, a test catalytic reaction (styrene hydrogenation) was performed to probe the reactivity of these RuNPs.

The ligand ICy.(p-tol) NCN was readily obtained from the reaction of 1,3-dicyclohexylimidazolidene (ICy) and di-p-tolylcarbodiimide (p${ }^{\text {tol) }} \mathrm{NCN}$ ) (see ESI $\dagger$ ). Further details on the synthesis, characterization and coordination chemistry of this and other NHC-CNC ligands will be reported elsewere. ${ }^{7} \mathrm{Ru}$ nanoparticles were synthesized with this betaine adduct following our organometallic approach as previously described. ${ }^{4}$ A THF solution of the complex [Ru(COD)(COT)] was treated at room temperature (r.t.) under 3 bar $\mathrm{H}_{2}$ in the presence of ICy ${ }^{\text {(p-tol) }} \mathrm{NCN}$ (Scheme 1). Various ICy. ${ }^{\text {(p-tol) }} \mathrm{NCN} / \mathrm{Ru}$ ratios were applied, namely $0.1,0.2$ and 0.5 molar equivalent (equiv.) giving rise to RuNP samples called Ru-ICy.(p-tol) $\mathrm{NCN}_{0.1}, \mathrm{Ru}-\mathrm{ICy} \cdot{ }^{\text {(p-tol) }} \mathrm{NCN}_{0.2}$ and $\mathrm{Ru}-\mathrm{ICy}{ }^{\cdot(\mathrm{p}-\text { tol) }} \mathrm{NCN}_{0.5}$, respectively. In all the situations a black stable colloidal solution was obtained which was subsequently purified by precipitation in pentane, finally affording the RuNPs as a black 


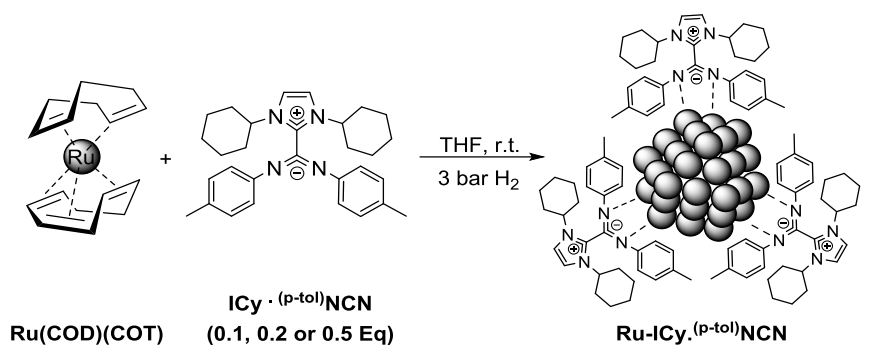

Scheme 1. Synthesis of ruthenium nanoparticles in the presence of ICy ${ }^{\text {(p-tol) }} \mathrm{NCN}$ as stabilizer.
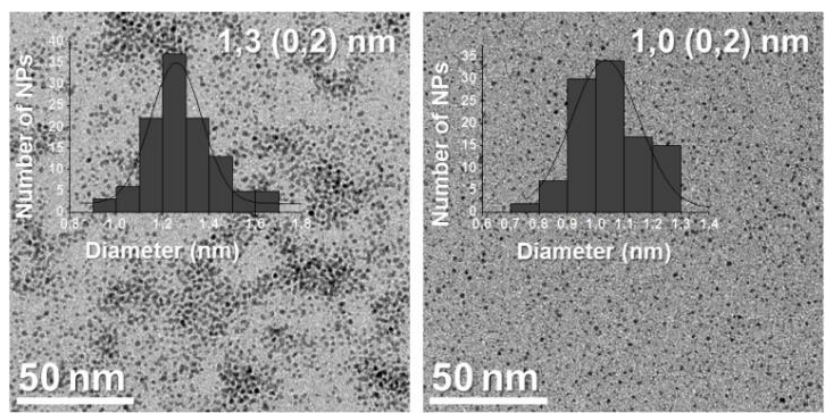

Figure 1. TEM images and the corresponding size histograms of Ru$\mathrm{ICy}^{\text {(p-tol) }} \mathrm{NCN}_{0.1}$ (left) and $\mathrm{Ru}-\mathrm{ICy}$ (p-tol) $^{\text {NCN }} \mathrm{N}_{0.2}$ (right).

powder. Transmission Electronic Microscopy (TEM) images and the corresponding size histograms of purified Ru-ICy.(p-tol) $\mathrm{NCN}_{0.1}$ and $\mathrm{Ru}_{-\mathrm{ICy}}{ }^{(\mathrm{p}-\mathrm{tol})} \mathrm{NCN}_{0.2} \mathrm{NPs}$ are given in Figure 1. As previously observed with NHC-carbenes and other stabilizers, ${ }^{5 a, 8}$ smaller NPs were formed when increasing the amount of ligand from 0.1 to 0.2 equiv. Indeed, in sample $\mathrm{Ru}-\mathrm{ICy}{ }^{(\mathrm{p}-\text {-tol) }} \mathrm{NCN}_{0.1}$, the $\mathrm{NPs}$ have a mean diameter of ca. $1.3(0.2) \mathrm{nm}$, whilst for $\mathrm{Ru}-\mathrm{ICy} \mathrm{y}^{(\mathrm{p}-\mathrm{tol})} \mathrm{NCN}_{0.2}$, they have a mean size of ca. $1.0(0.2) \mathrm{nm}$. Nevertheless, Ru-ICy.(p${ }^{\text {tol) }} \mathrm{NCN}_{0.5}$ NPs have also a mean diameter of ca. $1.0(0.2) \mathrm{nm}(\mathrm{S} 1$, $\left.\mathrm{ESI}^{\dagger}\right)$. These results indicate that the ligand concentration influences the NP mean diameter until a certain limit since adding more than 0.2 equiv. of betaine does not change the NP mean size. A similar behaviour was previously observed with a sulfonated diphosphine and explained as a steric hindrance phenomenon. ${ }^{9}$ In all cases the NPs are well-distributed on the TEM grid and display a relatively narrow size distribution. High Resolution Transmission Electron Microscopy (HRTEM) images of Ru-ICy. ${ }^{\left({ }^{\text {-tol })} \mathrm{NCN}_{0.1}\right.}$ (S2, $\left.\mathrm{ESI}^{\dagger}\right)$ revealed the presence of highly crystalline NPs retaining the hexagonal close packed (hcp) structure of bulk Ru. Fourier analysis applied to this image shows reflections to the (101), (101) and (100) atomic planes. Wide-Angle X-ray Scattering (WAXS) analysis performed on solid powder of $\mathrm{Ru} / \mathrm{ICy}{ }^{\left(\mathrm{p}^{-t o l}\right)} \mathrm{NCN}_{0.1}$ confirmed that these NPs are crystalline with a hcp structure. The Radial Distribution Function (RDF) analysis does not indicate oxidation, but shows metallic Ru NPs with a single domain and a coherence length close to ca. $1 \mathrm{~nm}$, which is in agreement with the HRTEM observations $\left(\mathrm{S} 3, \mathrm{ESI}^{\dagger}\right)$.

In order to understand the influence of ICy. ${ }^{\left({ }^{-t o l}\right)} \mathrm{NCN}$ on the size control during the RuNP preparation, we investigated its coordination at the metallic surface by NMR spectroscopy. Thus, the RuNPs were analyzed by magic angle spinning solid-state NMR (MAS-NMR) with and without ${ }^{1} \mathrm{H}-{ }^{13} \mathrm{C}$ cross-polarization (CP). CPMAS NMR spectra of $\mathrm{Ru}-\mathrm{ICy} \cdot{ }^{(\mathrm{p}-\mathrm{tol})} \mathrm{NCN}_{0.1}$ recorded on a purified $\mathrm{NP}$ sample and on the free $\mathrm{ICy} \cdot{ }^{(\mathrm{p}-\mathrm{tol})} \mathrm{NCN}$ ligand are presented together on Figure 2 for comparison purpose. As it can be seen, most of the ligand signals can be clearly identified on the spectrum of the NPs. Besides prominent signals corresponding to the p-tolyl methyl group centered at $c a .25 \mathrm{ppm}$, a broad resonance at $30 \mathrm{ppm}$ for the cyclohexyl substituents and a characteristic low field narrow peak at $60 \mathrm{ppm}$ attributed to the nitrogen-bound methyne group, the spectrum of the NPs displays a peak at ca. $120 \mathrm{ppm}$ for the aromatic imidazolium backbone and signals at ca. $130 \mathrm{ppm}$ for the aromatic rings of p-toluene. But, the signals for the imidazolium and carbodiimide quaternary $\mathrm{NCN}$ atoms, observed as singlets in the ${ }^{13} \mathrm{C}$ MAS-NMR spectrum of the free ligand at 144.7 and $150.1 \mathrm{ppm}$ respectively, are not observed. The lack of visibility of these two peaks could be attributed to a line broadening induced by coordination of the ligand at the metal surface, as previously observed with NHC-carbenes for example. ${ }^{3 a}$ Another hypothesis could be a decomposition of the betaine ligand during the formation of the NPs. Such a decomposition could cause the break of the NNC$C \mathrm{NN}$ bond and afford carbene and carbodiimide units.

In order to elucidate the nature of the molecules coordinated on the ruthenium surface, different experiments were carried out, such as the reaction of $\mathrm{Ru}-\mathrm{ICy} \cdot{ }^{(p-t o l)} \mathrm{NCN}_{0.1}$ with octanethiol or the oxidation of $\mathrm{Ru}-\mathrm{ICy}{ }^{\cdot(\mathrm{p}-\mathrm{tol})} \mathrm{NCN}_{0.2}$. Given that thiols are well-known as strong coordinating ligands at metal surfaces, ${ }^{10}$ this experiment aimed at releasing the coordinated molecules from the NP surface, and detect them by ${ }^{1} \mathrm{H}$ and ${ }^{13} \mathrm{C}\left\{{ }^{1} \mathrm{H}\right\}$ solution NMR. A suspension of Ru-ICy. (p${ }^{\text {tol) }} \mathrm{NCN}_{0,1}$ in $\mathrm{C}_{6} \mathrm{D}_{6}$ was treated with 10 equiv. of octanethiol and then heated at $65^{\circ} \mathrm{C}$. Spectra recorded after $24 \mathrm{~h}$ of reaction $\left(\mathrm{S} 4, \mathrm{ESI}^{\dagger}\right) \mathrm{did}$ not show the presence of free ICy.(p-tol) $\mathrm{NCN}$ ligand in the liquid phase. However, di-n-octyl disulfide was observed (triplet and quintuplet at 2.55 and $1.61 \mathrm{ppm}$, respectively). The presence of disulfide was attributed to the catalytic oxidation of octanethiol into disulfide at the RuNP surface as previously observed during the synthesis of RuNPs stabilized with an excess of octanethiol. ${ }^{11}$ These results indicated first that the $\mathrm{ICy}^{\cdot(\mathrm{p}-\mathrm{tol})} \mathrm{NCN}$ ligand was not displaced at all from the NP surface. Second, octanethiol could react at the metal surface to form disulfide, showing that $\mathrm{Ru}$ sites remain available for its coordination and reaction. As another attempt to decoordinate the molecules from the metallic surface, the Ru-ICy.(p${ }^{\text {tol) }} \mathrm{NCN}_{0.2}$ NPs were treated under oxidative conditions. Such reaction test was previously applied with diphosphine-stabilized RuNPs for which it was not possible to prove by direct solution NMR studies the coordination of the phosphine ligands while free phosphine oxide was released and became visible after oxidation. ${ }^{12}$ For that purpose, a suspension of the NPs in EtOH was refluxed

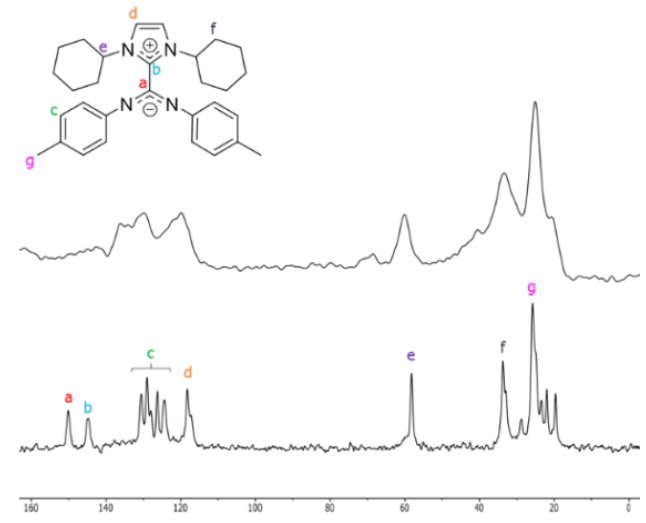

Figure 2. Bottom: CP-MAS ${ }^{13} \mathrm{C}\left\{{ }^{1} \mathrm{H}\right\}$ NMR spectrum of betaine adduct ICy.(p-tol) NCN. Top: CP- MAS ${ }^{13} \mathrm{C}\left\{{ }^{1} \mathrm{H}\right\}$ NMR spectrum of

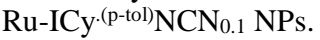


under air at $80{ }^{\circ} \mathrm{C}$ for $4 \mathrm{~h}$. After this treatment we observed the release of molecules in solution that correspond to $\mathrm{ICy}^{\text {(p-tol) }} \mathrm{NCN}$ ligand, as determined by ElectroSpray Ionization Mass Spectrometry (ESI-MS) (S5, ESI ${ }^{\dagger}$ ). Thus, it can be assumed that the betaine ligand remains intact when coordinated at the metal surface. As a consequence, the absence of signals at $144 \mathrm{ppm}$ and $150 \mathrm{ppm}$ in the ${ }^{13} \mathrm{C}$ MAS-NMR spectrum of the NPs is most probably due to a line broadening arising from the coordination of the ligand at the ruthenium surface through the NCN nitrogen atoms which may be reinforced by the sigma-donor ability of the carbene moiety. This coordination mode could be evidenced by doing a blank test consisting in using the carbodiimide ligand (p-tol) $\mathrm{NCN}$ ( 0.2 equiv.) to prepare RuNPs. In these conditions, ca. $1.6(0.4) \mathrm{nm}$ were obtained $\left(\mathrm{S} 6, \mathrm{ESI}^{\dagger}\right)$, thus proving the ability of the $\mathrm{NCN}$ motif to coordinate at the metallic surface.

The next step was to probe the surface state of the particles by measuring their capacity to bind $\mathrm{CO}$ by Fourier transform infrared (FT-IR) and MAS ${ }^{13} \mathrm{C}\left\{{ }^{1} \mathrm{H}\right\}$ and CP MAS ${ }^{13} \mathrm{C}\left\{{ }^{1} \mathrm{H}\right\} \quad$ NMR. Interestingly, FT-IR spectra of purified NPs Ru-ICy ${ }^{\left({ }^{(p-t o l)}\right.} \mathrm{NCN}_{0.1}$ and $\mathrm{Ru}-\mathrm{ICy}{ }^{(\mathrm{p}-\mathrm{tol})} \mathrm{NCN}_{0.2}$ showed a band displaying the characteristic of a CO absorption between 1900 and $2000 \mathrm{~cm}^{-1}$ before their exposure to $\mathrm{CO}$. Moreover, for the $\mathrm{Ru}-\mathrm{ICy} \cdot{ }^{(\mathrm{p} \text {-tol) }} \mathrm{NCN}_{0.1}$ sample, the intensity of the band did not change after $\mathrm{CO}$ exposure ( 1 bar; r.t.), suggesting that the NP surface was already saturated by $\mathrm{CO}$ after synthesis. RuICy.(p-tol) $\mathrm{NCN}_{0.2}$ showed a different band intensity suggesting that in this case the Ru surface was not totally covered by CO, (S7 and S8, $\left.\mathrm{ESI}^{\dagger}\right)$. Then, the question was: where does $\mathrm{CO}$ come from? Considering the reaction conditions for the RuNPs synthesis, the only source of CO could be THF, through a decarbonylation process which would release propane. We thus analyzed the composition of the reaction sky resulting from the NP synthesis mixture by gas phase NMR and Mass Spectrometry (MS). After the synthesis of RuICy.(p-tol) $\mathrm{NCN}_{0.1}$, we observed two peaks at 0.75 and $1.20 \mathrm{ppm}$ by gas phase NMR corresponding to propane (S9, $\left.\mathrm{ESI}^{\dagger}\right)$. By MS both compounds, $\mathrm{CO}$ and propane, were detected $\left(\mathrm{S} 10, \mathrm{ESI}^{\dagger}\right)$. In addition, following a previously reported procedure ${ }^{13}$ the RuNPs were reacted with 2-norbornene and the amount of norbornane formed was measured by G.C. analysis (see ESI $\dagger$ ). Ru-ICy.(p-tol) $\mathrm{NCN}_{0.1}$ and $\mathrm{Ru}-$

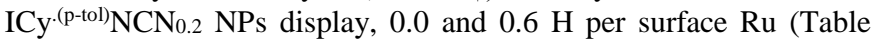
$\mathrm{S} 1, \mathrm{ESI}+)$; These values are lower than usual ones for other RuNPs and evidence elimination of hydrides from the surface probably due to a full or partial coverage of the $\mathrm{Ru}$ surface by $\mathrm{CO}$, respectfully. Altogether, these data demonstrate that $\mathrm{CO}$ is coordinated at the RuNP surface, as the result of a decarbonylation process of THF used as solvent during their synthesis, hence evidencing the high surface reactivity of these NPs. The MAS ${ }^{13} \mathrm{C}\left\{{ }^{1} \mathrm{H}\right\}$ NMR spectrum of solid samples of Ru-ICy ${ }^{\text {(p-tol) }} \mathrm{NCN}_{0.1}$ NPs exposed to 1 bar of ${ }^{13} \mathrm{CO}$ at r.t. (Figure 3 left) displays two signals attributed to adsorbed CO molecules: a broad peak at $\delta \sim 229$ ppm that can be assigned to ${ }^{13} \mathrm{CO}$ coordinated in a bridging mode $(\mathrm{CO} b)$ and a sharp resonance at $\delta=199 \mathrm{ppm}$ due to ${ }^{13} \mathrm{CO}$ coordinated in a terminal mode $(\mathrm{CO} t)$. From previous works, bridging $\mathrm{CO}$ molecules are expected to be coordinated onto faces and terminal ones onto corners and edges. ${ }^{13}$ Moreover, the presence of spinning bands (*) suggests that $\mathrm{CO} t$ are static on the surface. The limitation of CO mobility on the metallic surface can be explained by the coordination of betaine ligands in close proximity of the ruthenium sites where $\mathrm{CO}$ are coordinated. On the CP MAS ${ }^{13} \mathrm{C}\left\{{ }^{1} \mathrm{H}\right\}$ NMR spectrum (S11, ESI ${ }^{\dagger}$ ), the intensity of the signal for bridging $\mathrm{CO}$ is decreased compared to that of the terminal ones. This means that bridging $\mathrm{CO}$ groups are not affected by cross polarization from close hydrogen carriers while terminal $\mathrm{COs}$ are. It can be thus assumed that bridging $\mathrm{CO}$ groups are not located in the vicinity of hydrogen carriers (namely, betaine ligands)

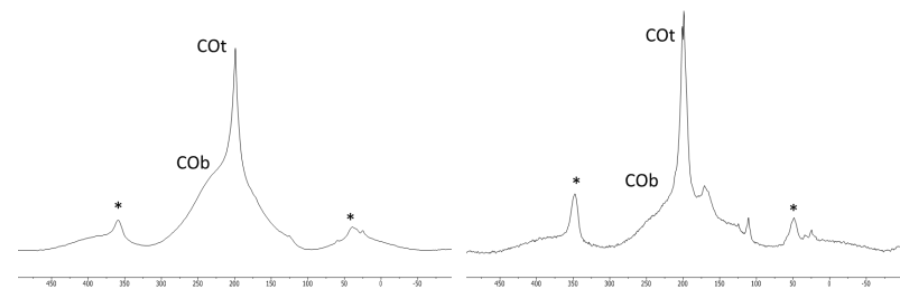

Figure 3. MAS ${ }^{13} \mathrm{C}\left\{{ }^{1} \mathrm{H}\right\}$ NMR spectra of Ru-ICy'(p-tol) $\mathrm{NCN}_{0.1}$ (left) and $\mathrm{Ru}-\mathrm{ICy} \cdot{ }^{(\mathrm{p}-\text {-tol) }} \mathrm{NCN}_{0.2}$ after exposure to ${ }^{13} \mathrm{CO}$ (1 bar, 20h, at r.t.).

while $\mathrm{CO} t$ are. This observation is in agreement with the lack of for mobility observed terminal $\mathrm{CO}$ which can result from the nearby coordination of betaine ligands thus limiting their moving. As the main difference between the two RuNP samples is the decrease in size with increased ligand quantity (ca.1.0 nm for Ru-ICy.(p${ }^{\text {tol) }} \mathrm{NCN}_{0.2}$ against ca. $1.3 \mathrm{~nm}$ for $\mathrm{Ru}-\mathrm{ICy} \cdot{ }^{(\mathrm{p}-\text { tol) }} \mathrm{NCN}_{0.1}$ ), a plausible explanation could be that the coordination of $\mathrm{CO}$ in a bridging mode is hindered by a lack of available faces on the surface for the smaller

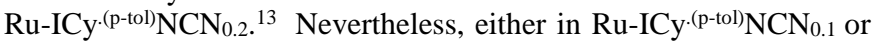
$\mathrm{Ru}-\mathrm{ICy}$ (p-tol) $^{\mathrm{NCN}_{0.2}} \mathrm{NPs}, \mathrm{CO}$ is mainly coordinated in terminal mode. This can result from the very small size of the NPs, where the face surfaces is limited and most ruthenium atoms concentrate on edges and apexes, where coordination in a terminal mode is more favorable.

The small size and specific electronic configuration of MNPs make them interesting systems for many catalytic reactions. In particular RuNPs are able to catalyze both the hydrogenation of alkenes and arenes under mild conditions while molecular complexes are just active in alkene hydrogenation. ${ }^{14}$ Styrene hydrogenation can thus be applied as a model reaction to probe the surface reactivity of MNPs because it offers the possibility of measuring the selectivity between vinyl and aromatic hydrogenation. Thus, we investigated the performance of Ru-ICy.(p-tol) NCN NPs in styrene hydrogenation in mild reaction conditions. Table 1 reports the results obtained in styrene hydrogenation with $\mathrm{Ru}-\mathrm{ICy} \cdot{ }^{(\mathrm{p}-\mathrm{tol})} \mathrm{NCN}_{0.1}$ and $\mathrm{Ru}-\mathrm{ICy} \cdot \mathrm{p}^{\text {(p- }}$ ${ }^{\text {tol) }} \mathrm{NCN}_{0.2}$ at r.t. in THF. Ru-ICy.(p-tol) $\mathrm{NCN}_{0.1}$ hydrogenates styrene first to ethylbenzene and then to ethylcyclohexane. The Ru-ICy.(p${ }^{\text {tol) }} \mathrm{NCN}_{0.1}$ catalyst allowed the total hydrogenation of styrene into 24h. While using Ru-ICy.(p-tol) $\mathrm{NCN}_{0.2}$ as nanocatalyst, the hydrogenation rate of the vinyl group remained similar to that of $\mathrm{Ru}-$ ICy.(p-tol) $\mathrm{NCN}_{0.1}$, the hydrogenation rate of the arene moiety decreased. The selectivity for vinyl hydrogenation was therefore lightly improved: $74 \%$ of the ethylbenzene was hydrogenated to ethylcyclohexane after $24 \mathrm{~h}$. As the presence of available faces are necessary to hydrogenate aromatic rings, ${ }^{15}$ these catalysis results corroborate the fact that less faces are present in $\mathrm{Ru}-\mathrm{ICy} \cdot{ }^{(\mathrm{p} \text {-tol) }} \mathrm{NCN}_{0.2}$ $\mathrm{NPs}$ than in $\mathrm{Ru}-\mathrm{ICy}{ }^{(\mathrm{p}-\mathrm{tol})} \mathrm{NCN}_{0.1}$ ones. This makes Ru-ICy.(p${ }^{\text {tol) }} \mathrm{NCN}_{0.2} \mathrm{NPs}$ to have a behavior in catalysis more similar to that of molecular complexes than to facetted nanoparticles. ${ }^{16}$

\section{Conclusions}

A betaine adduct of NHC and carbodiimide (NHC-NCN) has been identified as a new ligand capable of stabilizing very small $\mathrm{Ru}$ nanoparticles. Specifically, ICy. ${ }^{\left({ }^{\text {p-tol })}\right.} \mathrm{NCN}$ gave rise to ultra-small and crystalline Ru NPs. A clear relationship was observed between the ICy. ${ }^{(p-t o l)} \mathrm{NCN} / \mathrm{Ru}$ ratio used in the NP synthesis and the mean size of the obtained NPs. In particular ca. $1.3 \mathrm{~nm}$ NPs were produced when 0.1 equiv. of ICy. ${ }^{(p-t o l)} \mathrm{NCN}$ per $\mathrm{Ru}$ were used and ca. $1.0 \mathrm{~nm}$ NPs in the case of 0.2 equiv. This difference in size leads to different 
Table 1. Hydrogenation of styrene with Ru-ICy'(p-tol) NCN NPs.

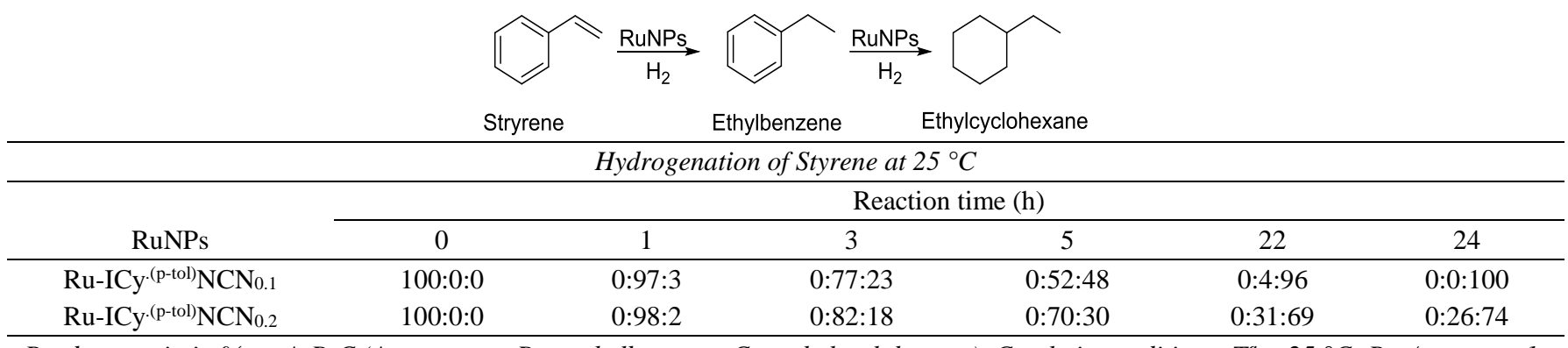

Products ratio in \% to A:B:C (A = styrene; $B=$ ethylbenzene; $C=$ ethylcyclohexane $)$. Catalytic conditions: $T^{a}=25{ }^{\circ} C ;$ Rus $/$ styrene $\sim 1$ : 300; $\mathrm{Ru}_{\mathrm{s}} \sim 0,03 \mathrm{mmol} ; \mathrm{H}_{2}$ Pressure $=3$ bar. Conversion determined by G.C.

availabilities in terms of accessible faces at the metal surface, the smallest RuNPs displaying less faces as expected. This was evidenced by studying the coordination of $\mathrm{CO}$ as well as the hydrogenation of styrene as model substrate. While larger NPs (RuICy.(p-tol) $\mathrm{NCN}_{0.1}$ ) were able to transform totally styrene into ethylcyclohexane in mild reaction conditions, the smaller particles Ru-ICy.(p-tol) $\mathrm{NCN}_{0.2}$ NPs provided a partial selectivity into ethyl benzene. Such behaviour in catalysis is expected for molecular complexes but not for facetted RuNPs. Thus, even if they are not perfectly defined, these ca. $1 \mathrm{~nm}$ RuNPs display a reactivity at the frontier between the molecular and solid states. The next step would be to determine the nano-object size or ligand-covering at which the hydrogenation of the arene ring is totally blocked while keeping that of the external double-bond in styrene hydrogenation. Furthermore, decarbonylation of THF, the solvent used for the synthesis of these NPs was evidenced for the first time and is probably related to the high reactivity of these ultra-small RuNPs. This result makes these new RuNPs of interest for exploring other type of catalytic reactivity at the border of the molecular and the solid states.

The authors thank CNRS, UPS-Toulouse, INSA, CSIC, IIQ, Spanish and Regional Andalusian Governments (grants CTQ2012-30962 and FQM6276) and EU (ERC Advanced Grant, NANOSONWINGS 2009-246763) for financial support. We also thank V. Collière and L. Datas for TEM facilities (TEMSCAN, UPS), P. Lecante (CEMES, CNRS) for WAXS measurements, C. Bijani and Y. Coppel for NMR measurements, and A. Márquez Esteban for useful help on the synthesis of betaine ligand ICy.(p-tol) NCN.

\section{Notes and references}

${ }^{a}$ Laboratoire de Chimie de Coordination; CNRS; LCC; 205, Route de Narbonne, F-31077. Université de Toulouse, UPS, INPT, LCC, 31077 Toulouse, France. E-mail: karine.philippot@1cc-toulouse.fr

${ }^{b}$ Instituto de Investigaciones Químicas, CSIC - Universidad de Sevilla. C/ Américo Vespucio, 49, 41092 Sevilla, Spain. E-mail: campora@iiq.csic.es

c LPCNO; Laboratoire de Physique et Chimie des Nano-Objets, UMR5215 INSA-CNRS UPS, Institut des Sciences appliquées, 135, Avenue de Rangueil, F-31077 Toulouse, France. E-mail: chaudret@insatoulouse.fr

$\dagger$ Electronic Supplementary Information (ESI) available: [details of any supplementary information available should be included here]. See DOI: $10.1039 / \mathrm{c} 000000 \mathrm{x} /$
1 Nanoparticles. From Theory to Application ed. G. Schmid, WileyVCH, Weinheim 2004.

2 Term "size" refers to "mean diameter"

3 a) K. Philippot and B. Chaudret, C.R. Chimie 2003, 6, 1019. b) C. Vollmer, E. Redel, K. Abu-Shandi, R. Thomann, H. Manyar, C. Hardacre and C. Janiak, Chem. - A Eur. J. 2010, 16, 3849. c) D. Marquardt, C. Vollmer, R. Thomann, P. Steurer, R. Mülhaupt, E. Redel and C. Janiak, Carbon 2011, 49, 1326.

4 a) Organometallic Ruthenium Nanoparticles and Catalysis, K. Philippot, P. Lignier and B. Chaudret, in Ruthenium in Catalysis. Topics in Organometallic Chemistry, eds. Dixneuf, P. H., Bruneau, C.; Springer International Publishing 2014.b) P. Lara, K. Philippot, and B. Chaudret, Chem CatChem 2013, 5, 28.

5 a) P. Lara, O. Rivada-Wheelaghan, S. Conejero, R. Poteau, K. Philippot and Chaudret, Angew. Chem. Int. Ed. 2011, 50, 12080. b) P. Lara, A. Suárez, V. Collière, K. Philippot and B. Chaudret, Chem CatChem 2014, 6, 87.

6 L. Delaude, Eur. J. Inorg. Chem. 2009, 1681.

7 J. Cámpora et al., to be submitted.

8 M. Zahmakiran, K. Philippot, S. Özkar and B. Chaudret, Dalton Trans. 2011, 41, 590.

9 M. Guerrero, Y. Coppel, N. T. T. Chau, A. Roucoux, A. DenicourtNowicki, E. Monflier, H. Bricout, P. Lecante, K. Philippot, ChemCatChem, 2013, 12, 3802.

10 I. Favier, S. Massou, E. Teuma, K. Philippot, B Chaudret and M. Gomez, Chem. Comm. 2008, 3296.

11 C. Pan, K. Pelzer, K. Philippot, B. Chaudret, F. Dassenoy, P. Lecante and M.-J. Casanove, J. Am. Chem. Soc. 2001, 123, 7584.

12 J. García-Antón, M. R. Axet, S. Jansat, K. Philippot, B. Chaudret, T. Pery, G. Buntkowsky and H.-H. Limbach, Angew. Chem. Int. Ed. 2008, 47, 2074.

13 F. Novio, K. Philippot and B. Chaudret, Catal Lett 2010, 140, 1.

14 Nanoparticles and Catalysis, ed. D. Astruc, Wiley-VCH, Weinheim, 2008.

15 B. Hammer, O. H. Nielsen, J. K. Nørskov, Catal. Lett. 1997, 46, 31.

16 C.-S. Chena, J. H. Linb, H.-W. Chenc, C.-Y. Wang, Catal. Lett. 2005, 105, 149. 